\title{
Redefiniendo la profesión: los médicos de Barcelona en el nuevo milenio
}

\section{Re-defining the profession: Barcelona's physicians in the new millenium}

Josep A. Rodríguez

Equipo de Investigaciones en Profesiones y Organizaciones. Departament de Sociologia i AO. Universitat de Barcelona.

\section{Utilizando la reciente encuesta "Los médicos de Barcelona en el nuevo milenio" analizamos los procesos de adaptación de la profesión médica a las nuevas dinámicas sociales, así como a las fuerzas de las organizaciones, el estado y el mercado. Como parte de ese ajuste, la profesión se redefine como profesión de servicios, entre el Estado y el Mercado, con planteamientos organizativos nuevos, y reafirmado su orientación social.}

Palabras clave: profesión médica, nuevo milenio, adaptación, orientación social

\begin{abstract}
Using the recent survey "Barcelona's physicians in the new millennium", in this paper we study the processes of adaptation of the medical profession to the new social dynamics, as well as to the organization, state and market forces. As a part of this adjustment process, the medical profession redefines itself as a service profession, between the state and the market, with new organizational proposals and reaffirming its orientation to society.
\end{abstract}

Key words: medical profession, new millennium, adjustemt, social orientation
La profesión médica, al igual que los sistemas y profesiones sanitarias, se encuentra inserta en un conjunto de procesos de cambio que caracterizan a la sociedad del siglo XXI. Procesos de tipo social y cultural, organizativos, tecnológicos y políticos están suponiendo nuevos retos a la vez que nuevas oportunidades para la profesión y su futuro ${ }^{1,2,3,4}$.

De entre los procesos sociales debemos destacar los cambios en las estructuras poblacionales y de valores que van desde la incorporación de las mujeres a la universidad y a las profesiones (entre ellas a la medicina), a la gran ampliación de las clases medias enarbolando un nuevo sistema de valores, la configuración de sociedades donde el grupo de población de más de 75 años crece con velocidad, y la presencia creciente de nuevas poblaciones emigrantes,

\footnotetext{
Correspondencia:

Josep A. Rodríguez

Equipo de Investigaciones en Profesiones y Organizaciones Departament de Sociologia i AO. Universitat de Barcelona. e-mail: jarodriguez@ub.edu
}

con tipos y niveles de demanda diferentes de las poblaciones locales. Ello se traduce en la articulación tanto de nuevas ofertas profesionales y modelos de ejercicio como de nuevas demandas y nuevas formas de demanda (nuevas enfermedades, demanda de servicios adaptados a las características y valores poblacionales). Mientras que por un lado se refuerza la aproximación de lucha contra la enfermedad, por el otro se amplía el concepto de salud incorporando las dimensiones de calidad de vida y como resultado se genera una nueva demanda orientada más a la salud que a la mera ausencia de enfermedad.

Los procesos organizativos más relevantes están centrados en la creciente tensión entre los modelos organizativos y de gestión propios de la sociedad industrial (donde se conceptualizaba la sanidad en términos de proceso de producción) con modelos organizativos propios de la sociedad de servicios; la mercantilización de la salud, con el crecimiento del mercado de productos de salud (y de su correspondiente demanda) junto al crecimiento de proveedores sanitarios con formas organizativas de provisión de servicios 
marcadas por el beneficio; el dominio de ideologías economicistas en los modelos sanitarios; y la creciente importancia de los debates girando alrededor de la propiedad versus titularidad de las instituciones públicas y acerca de la gobernabilidad institucional.

Los procesos tecnológicos más destacados son la rápida configuración de una sociedad global con rápidos flujos de información y personas que acentúa, la trasmisión global de enfermedades y el impacto mediático creciente de las enfermedades (locales o globales); el aumento de la facilidad para obtener y trasmitir información a través de internet que redunda en un ciudadano consumidor con más conocimientos y más propenso al autocuidado a la vez que abre vías a nuevas formas de realización de la práctica médica basadas en la telemedicina y medicina virtual; así como los avances tecnológicos y robóticos que posibilitan también cambios en ciertos procedimientos médicos (cirugía, trasplantes, etc) y los avances en el conocimiento del genoma humano abriendo la puerta a la biomedicina.

Estos procesos colocan a la profesión ante demandas y presiones, a veces contradictorias, de cambio. Se camina hacia un nuevo pacto social (entre la profesión y la sociedad) que asegure la adecuada respuesta a las nuevas demandas sociales y de los ciudadanos en el marco de las tendencias reguladoras y de reducción de costes. La profesión se enfrenta a formas de ejercicio en marcos organizativos complejos dominados por un creciente conflicto entre definiciones contradictorias de beneficio y de dinámica organizativa. La emergencia de nuevos mercados y nuevas formas de demanda (consultor de salud) son también clave en la configuración de ese nuevo pacto social. Todo ello plantea la necesidad de que la profesión médica disponga de un proyecto de futuro estructurado que le permita participar de forma coherente en los procesos políticos de redefinición de la medicina y la sanidad.

En las páginas siguientes se recogen brevemente algunos de los resultados generales de la reciente encuesta Els Metges de Barcelona en el Nou Mil.leni ${ }^{5}$ que nos permiten visionar algunos de los procesos de ajuste al medio cambiante así como algunas de sus posiciones y respuestas ante los retos del futuro.

\section{NUEVA PROFESIÓN PARA NUEVA SOCIEDAD}

Debemos empezar señalando dos niveles de transformaciones afectando a la profesión: los cambios sociales y demográficos de la profesión en si misma, y los cambios en su entorno. La conjunción de ambos conduce a la reconfiguración tanto de la profesión como de su oferta y demanda de servicios.
La entrada numéricamente importante de nuevos profesionales en las últimas décadas tiene como resultado la configuración de una profesión estructuralmente nueva, relativamente joven y con un alto componente femenino. Su media de edad es de 46 años y la mitad de los profesionales tienen a su vez menos de 46 años. De hecho una cuarta parte de los profesionales llevan menos de 11 años ejerciendo (y casi la mitad de la profesión tiene una vida profesional de menos de 20 años).

Una de las transformaciones más importantes y recientes en las profesiones y sus mercados de servicios es la creciente participación de las mujeres. Este es un elemento demográfico que está teniendo un gran impacto tanto en la estructura como en la dinámica profesional, y que previsiblemente puede afectar su futuro. La composición por género de la profesión barcelonesa es una muestra de este proceso: el $40 \%$ de los profesionales actuales son mujeres. Su participación en la profesión ha ido aumentando rápidamente con los años siendo especialmente destacada en las nuevas cohortes de profesionales (llegando a representar el $71 \%$ en el grupo entrando en la profesión).

Un elemento que ha caracterizado a las profesiones en las últimas décadas ha sido su progresiva especialización, en respuesta tanto a la expansión de sus mercados como a los avances en el conocimiento. Las profesiones se convierten en espacios complejos que articulan un número creciente de especializaciones. Este fenómeno es especialmente agudizado en la Medicina y tiene como resultado que la gran mayoría de los profesionales (70\%) esté especializado. Las mujeres profesionales destacan por su mayor especialización en Medicina General, mientras que los varones se concentran más en Especialidades Médicas y Especialidades Quirúrgicas.

La dimensión familiar de la Medicina, parentesco médico, siempre ha jugado un papel crucial en la profesión tanto en su dimensión socializadora como en la creación de identidad. En las nuevas cohortes profesionales dominan las relaciones de parentesco en la misma generación (esposos/as, hermanos, primos), mientras que en los mayores de 50 años dominan las relaciones de parentesco con generaciones anteriores (padres, tíos, abuelos). Es importante destacar los altos niveles de matrimonios entre profesionales (casi una cuarta parte de los profesionales), especialmente en las nuevas generaciones, configurando lo que podríamos llamar "dual-doctor families".

Históricamente la profesión ha jugado un papel destacado como espacio de reproducción para los estratos altos de la sociedad y también como vehículo de movilidad social. En la actualidad ese papel 
ha quedado limitado y se consolida como profesión de clase media. Este cambio está produciendo algunos problemas derivados de la disonancia entre la procedencia social, las expectativas de ascenso social y la realidad. Casi la mitad de los profesionales no ha alcanzado (todavía) la posición social que esperaba cuando entró en la profesión. Esta situación es especialmente notable en las mujeres profesionales, muchas de las cuales (una cuarta parte) dicen haber descendido socialmente.

Paralelamente, y en respuesta a una sociedad cada vez más rica donde se amplía el concepto de salud, la profesión amplía su visión de servicio y reconceptualiza las relaciones con los pacientes. Esta reconceptualización se articula en dos grandes aproximaciones al ejercicio profesional: por un lado una aproximación clásica de medicina "reparadora" y por otro lado una medicina más orientadora y responsable global del paciente. Los médicos manifiestan que la mayoría de sus pacientes (66\%) esperan de ellos una medicina reparadora pero preferirían que les demandaran una medicina más orientadora/total (59\%). Es decir, los pacientes esperan una medicina técnica y mecánica que solucione sus problemas y los profesionales, sin restar importancia a su acción curadora, preferirían ofrecer una medicina que asumiera la salud global del ciudadano.

Al mismo tiempo, se amplía también la demanda, y legitimidad social, de medicinas complementarias ${ }^{6,7}$. Una parte de los profesionales responde a estas nuevas demandas incorporando esos conocimientos en el ejercicio. Aunque el porcentaje de profesionales que utilizan sistemáticamente las medicinas complementarias es bajo, existe un número creciente que recurre a ellas (Acupuntura, Naturopatía y Homeopatía) de forma ocasional creando un nuevo nicho en el mercado con un nuevo producto médico. La integración (ocasional o sistemática) de la medicina complementaria en el ejercicio profesional configura un espacio profesional nuevo en el mercado, ocupado preferentemente por mujeres, jóvenes y no especialistas.

Recientes transformaciones en las sociedades ricas están dando lugar a nuevos cambios en el entorno social, con la presencia de nuevos problemas sociales y crecientes dificultades de comunicación con ciertos sectores de la población, que están suponiendo una importante presión adicional sobre los médicos barceloneses. La configuración de una demanda cada vez más compleja se traduce en un nivel creciente de esfuerzos adicionales. La diversidad de la composición social y cultural supone presiones asistenciales de tipo social y cultural por un lado y de tipo médico por otro. La base del sistema sanitario (fundamentalmen- te las profesionales, la asistencia primaria, los médicos generalistas, y los asalariados) es la que hace frente a las presiones de tipo social y cultural. Las nuevas problemáticas médicas recaen fundamentalmente en los especialistas y el sistema hospitalario. Evitar que esos esfuerzos adicionales se conviertan en tensiones requiere de iniciativas por parte de los sistemas sanitarios y de la profesión para adaptarse y atender esa demanda compleja de forma estandarizada.

Los cambios demográficos configuran una profesión de servicios para una sociedad rica. Es una profesión joven, con una creciente participación femenina, y de clase media, que esta cada vez más orientada a servicios de bienestar que amplían la orientación curativa clásica. Todo ello al mismo tiempo que nuevos procesos sociales y de salud presionan a los profesionales y al sistema sanitario hacia modelos y aproximaciones de lucha contra la enfermedad. El futuro de la profesión parece marcado por la tensión entre esos dos modelos de medicina.

\section{ENTRE EL ESTADO Y EL MERCADO}

El ejercicio de la profesión a principios del siglo XXI es un sistema complejo resultado en gran medida de procesos históricos y de la adaptación de la profesión a los cambios sociales y sanitarios ${ }^{8,9}$. La configuración y desarrollo del sistema sanitario ha llevado a la profesión a la articulación de una práctica compleja como forma de adaptación y de mantenimiento de las características básicas como profesión. Ello la lleva a posicionarse entre el estado y el mercado, con una lógica y racionalidad (organizativa y de servicios) propia ${ }^{10-14}$

Una de las características base de nuestro sistema sanitario es la articulación de un modelo de sanidad que combina la oferta pública con la oferta privada de servicios. En la actualidad la mayoría (63\%) de los profesionales barceloneses considera que la actual organización sanitaria con dominio público, y con espacio privado, es adecuada y deseable. Es un modelo sanitario especialmente defendido por las nuevas generaciones profesionales (y por las mujeres). Y al valorar y comparar el sistema público con el privado, la visión general es que el sector público ofrece un mejor producto (con mayor calidad técnica y clínica) mientras que el sector privado ofrece un mejor servicio (especialmente posibilitando una mejor relación médico-paciente).

Y uno de los elementos más característicos de la profesión médica barcelonesa es el hecho de que una gran parte de los profesionales (el 41\%) combine varias actividades profesionales. Es importante destacar que 
la combinación de actividades (y de espacios asistenciales) se ha convertido en un elemento fundamental de la carrera profesional, aumentando progresivamente con la edad. Las mujeres (más jóvenes de media) tienen básicamente una sola actividad, mientras que la mitad de los varones tiene al menos dos actividades. Se articula un modelo de ejercicio profesional donde el 59\% de los médicos sólo tiene un ejercicio (mayoritariamente en forma de empleo, normalmente público) y el $41 \%$ compagina al menos dos ejercicios donde se combinan ámbitos y formas de practicar.

La actividad principal está dividida entre el espacio hospitalario y el no-hospitalario, y la segunda actividad se realiza mayoritariamente en el espacio no-hospitalario. El ejercicio principal se realiza fundamentalmente como empleado en organizaciones sanitarias (mayoritariamente públicas). A pesar del dominio, y presión, creciente de la medicina organizada, todavía casi una quinta parte de los profesionales ejercen su actividad principal como profesionales liberales. Es importante notar la diferencia entre géneros: las mujeres tienden a ejercer en el espacio no-hospitalario y fundamentalmente público, mientras que los varones lo hacen más en el hospitalario.

Debemos resaltar también como elemento destacado de la vida profesional, la relativa estabilidad organizativa y su gran dedicación al ejercicio, con jornadas laborales superiores a la media laboral. Los médicos barceloneses llevan una media de 12 años en sus trabajos actuales (principal y secundario) y por término medio dedican una media de 46 horas semanales a su trabajo.

Esa posición intermedia entre el estado y el mercado, entre los espacios organizativos complejos y los espacios más individuales, la lleva a potenciar sistemas relacionales que superan las limitaciones organizativas y del mercado y que aseguren un cierto control sobre la oferta y demanda profesional ${ }^{15}$. Por ello, y a pesar de la importancia de la medicina organizada, y los sistemas relacionales basados en la organización, las relaciones entre colegas (de asesoramiento, consulta y colaboración) son fundamentales en el ejercicio profesional y configuran una forma de ejercicio que transciende y complementa a los modelos de práctica regulados por la organización. De hecho se configuran dos modelos de relación: uno dominado por el espacio organizativo (donde son dominantes las relaciones de asesoramiento y consulta) y otro que se extiende hacia la profesión en su conjunto (aquí dominan las relaciones de confianza y solicitud de ayuda ante situaciones graves). Estos dos espacios relacionales son los utilizados para responder adecuadamente a casos complejos: una parte de los profesionales recurre fundamental- mente a la organización, mientras que otra parte recurre a su sistema de relaciones a través de la profesión.

Ello facilita, a pesar del dominio de la medicina organizada, que los profesionales y la profesión todavía mantengan una amplia capacidad de generación directa de demanda de sus servicios: casi mitad de los pacientes atendidos o bien les llegan directamente o bien a través de otros colegas. Todavía mantienen una posición importante en el proceso de creación de demanda y oferta de sus servicios profesionales. Ello pone de manifiesto que, a pesar del dominio de la medicina organizada y del paciente organizativo, todavía a los ojos de muchos pacientes lo importante de la sanidad es el médico, y que los profesionales son todavía capaces de liderar/diseñar el producto-medicina y frenar su integración y difuminización en la organización y el producto organizado manteniendo una cuota importante de mercado individual (ya sea mercado libre o con pacientes asegurados).

A pesar de la fortaleza de las dinámicas organizativas, y del Estado y el mercado, la profesión médica es capaz de articular una práctica compleja entre ambos espacios trascendiendo las lógicas organizativas, y de mantener una cierta influencia sobre su oferta y demanda de servicios.

\section{EN UNA MEDICINA ORGANIZADA}

La configuración de sistemas sanitarios y médicos complejos, con un peso dominante de las grandes organizaciones, pone sobre el tapete la tensión entre los procesos de desprofesionalización y/o proletarización ${ }^{16-19}$ (estandarización, asalarización, pérdida de control) y la capacidad de la profesión para adaptarse y a la organización y mantener parte de la autonomía que la caracteriza como profesión ${ }^{20,21,22}$ y le permite mantener su identidad.

La creciente complejidad de las estructuras sanitarias y del propio ejercicio médico lleva aparejada la tendencia a la estandarización y a la "rutinización" como mecanismos de reducción de la incertidumbre a la vez que de mejora de la calidad asistencial. El resultado es que dos tercios de las decisiones que toman diariamente los profesionales son de tipo rutinario, especialmente en el ámbito nohospitalario, y el hecho de que en situaciones complejas las decisiones se toman a menudo en solitario (42\%) aunque la mayoría de los profesionales (81\%) preferirían tomarlas en equipo.

En la configuración de los ingresos de los profesionales convergen dos fuerzas a menudo contradictorias: el hecho de ser expertos con un alto nivel de formación (que tendería a ingresos altos en sociedades del cono- 
cimiento) y el dominio público del mercado y espacio profesional (que supone topes salariales y que reduce la extensión del mercado privado). El resultado, a pesar de que muchos médicos combinan varias prácticas profesionales, es que casi la mitad (47\%) de los profesionales tienen unos ingresos mensuales medios entre 300 y 500 mil pesetas mientras que el $29 \%$ no llega a las 300 mil, destacando la existencia de situaciones económicas precarias a la entrada de la profesión (entre los jóvenes, mujeres y no especialistas).

La autonomía ha sido históricamente uno de los baluartes de la profesión médica ${ }^{23,24}$. En términos globales, el grado de libertad que los profesionales barceloneses tienen para tomar decisiones es alto. Los profesionales mantienen altos niveles de control en el espacio técnico diagnóstico-terapéutico pero bastante menos control e influencia sobre la organización social de su trabajo. La tecnología ha permitido mantener y aumentar la autonomía en el centro del ejercicio profesional pero no ha podido frenar el declive progresivo de la influencia de los profesionales en la organización y dinámica social de su práctica (el espacio organizativo y político). Es decir, la tecnología (como expresión máxima de cientificidad) se convierte en clave para el mantenimiento y aumento de la autonomía técnica de la profesión. En ese sentido, el control sobre la tecnología es el vehículo de independencia profesional.

La tecnología es un elemento clave en la profesión. Su utilización ha supuesto (y supondrá en el futuro) una mejora sustancial en su capacidad médica (mejorando su capacidad de diagnóstico y tratamiento). Reafirma la condición científica de la profesión y la condición de experto de los profesionales. Sin embargo esas tecnologías no han facilitado ni mejorado (todavía) la relación de los profesionales con sus pacientes y clientes. Posiblemente el uso creciente de las tecnologías domina el ejercicio profesional de tal forma que la relación con el paciente se convierte progresivamente en un espacio de aplicación de tecnologías y limita la comunicación con el paciente.

Además, el uso de las tecnologías, especialmente las más avanzadas y costosas, generan dilemas y disyuntivas sociales y éticas. La mitad de los profesionales aboga por ofrecer todos los servicios asistenciales aunque estableciendo ciertas prioridades en los menos esenciales, una cuarta parte defiende un nivel de restricción mayor, y la otra cuarta parte aboga por no poner ningún tipo de limitaciones. Este tipo de situaciones sitúa a los profesionales entre los pacientes y el sistema y organización. La respuesta es una situación intermedia que asegure tanto el bienestar del paciente como la supervivencia de la organización.
La valoración y prestigio social son fundamentales para el mantenimiento de ciertos niveles de autonomía ${ }^{25-27}$,tanto como para asegurar la satisfacción e identidad profesional. En este sentido es interesante notar que la profesión está dividida prácticamente por la mitad en su percepción de su imagen pública. Un poco más de la mitad de los médicos barceloneses (51\%) no cree que la opinión pública tenga una buena imagen de ellos y el $49 \%$ cree que sí. De hecho, en general los profesionales se sienten bien valorados por sus pacientes y sus colegas, pero en cambio mal valorados por las organizaciones en que prestan sus servicios y por la sociedad en general. Posiblemente la difuminización de sus contribuciones individuales en un producto y organización complejos reduce su visibilidad y como consecuencia su valoración social. Como resultado una parte sustancial $(40 \%)$ de los médicos sienten que su profesionalidad y contribución no son suficientemente valoradas y compensadas por la sociedad (y un tercio adicional está resignado ante dicha valoración).

Pero a pesar de ello, los profesionales están bastante satisfechos son su profesión, y de hecho la mayoría $(78 \%)$ volvería a entrar en la misma (aunque un tercio con algunas dudas). A pesar de este alto nivel de satisfacción e identificación, los profesionales se dividen prácticamente por la mitad con respecto a si les gustaría o no que un familiar hiciese la carrera de medicina. Las dudas acerca de la medicina como espacio de profesionalización de sus hijos están obviamente relacionadas con los problemas en la organización social de su trabajo que limitan bastante las posibilidades de realización de una práctica excelente (retribuciones insuficientes, falta de incentivos, expectativas profesionales no satisfechas, y precariedad laboral). Por ello cuando definen su trabajo ideal acentúan la importancia de incrementar el control sobre la oferta profesional que asegure unas retribuciones adecuadas, una mayor capacidad de decisión sobre los ritmos de trabajo, y poder asegurar que su trabajo contribuye a la calidad de vida de las personas.

Sin duda, la profesión médica se encuentra sometida a fuerzas de desprofesionalización que resultan en pérdidas de control sobre la organización social de su ejercicio y de visibilidad y valoración pública. Pero, y a pesar de ello, es capaz de mantener altos niveles de control y autonomía sobre el corazón de la práctica y mantener un alto nivel de identificación y satisfacción con la profesión.

\section{REDEFINIENDO LA PROFESIÓN}

La adaptación de la profesión a los cambios de la 
sociedad y a las dinámicas organizativas y de los mercados se enmarca en la redefinición de la profesión, tanto en su modelo de ejercicio como de organización como colectivo así como en su contribución y papel social ${ }^{28,29}$.

Algo que ha distinguido históricamente a los profesionales, y específicamente a la profesión médica, de otros colectivos ocupacionales su capacidad de articular una visión propia de su práctica. La definición de profesión se convierte en un elemento central en los procesos de diseño de las estructuras y dinámicas del sistema y las organizaciones sanitarias. El ideal de ejercicio profesional deja de ser la práctica individual en solitario (que había definido históricamente a la profesión) y se centra ahora en los pequeños grupos de profesionales, frente al modelo dominante de medicina altamente organizada. Los pequeños grupos profesionales, todavía en espacios de propiedad y financiación pública pero con gestión más profesional o de tipo privado, se consolidan como la alternativa organizativa ideal para a la vez que hacer frente a las demandas sanitarias más complejas, hacerlo de la forma más eficiente (superando las limitaciones de las grandes organizaciones complejas).

El modelo de ejercicio ideal apoyado mayoritariamente por los profesionales es un modelo combinatorio. Es decir, que combine y compagine la práctica en el sector público con el privado, en el espacio hospitalario con el no-hospitalario, y como empleado a la vez que como profesional liberal. Para la mayoría de los profesionales la combinación de ámbitos y estructurales asistenciales asegura la maximización tanto de la calidad asistencial como de la calidad de las relaciones con los pacientes a la vez que su capacidad de toma de decisiones.

La mayoría de los profesionales participaría activamente en el cambio del sistema organizativo actual, ya sea mejorándolo o incorporando fórmulas que aumentasen la responsabilidad y capacidad de gestión de los profesionales. Es lógico que, dado que una parte sustancial de los problemas profesionales están relacionados con la organización de su trabajo, los profesionales estén muy interesados en configurar formas de ejercicio más adecuadas a la lógica de la demanda y oferta de sus servicios profesionales.

En los profesionales del siglo XXI se reafirma la visión de servicio y orientación al bienestar de los demás como los componentes principales del ejercicio de la medicina ${ }^{30,31}$. Obviamente ello es el reflejo de las visiones dominantes en la sociedad con respecto a la profesión médica, de la cual se espera siempre que trabaje para el bienestar colectivo. La clara orientación de servicio hacia el bienestar de los pacientes y la sociedad va más allá de la respuesta a las demandas puntuales (con problemas y necesidades) y articula un proyecto de bienestar para la comunidad. De hecho la visión dominante de la profesión es como actividad orientada a la ayuda a los demás. Esa orientación, claramente altruista, es todavía la razón y visión dominante al entrar en la profesión.

El ejercicio profesional es por tanto más que un trabajo, y de hecho la gran mayoría de los profesionales creen que el ejercicio requiere de una dedicación que trasciende los límites de una jornada laboral (ya sea con dedicación o total o más extensa que la jornada laboral media). En su orientación al bienestar y la ayuda a los demás trasciende las lógicas, y las jurisdicciones, de los mercados y organizaciones. De ahí que la excelencia profesional y el prestigio profesional, debería basarse en la ética, el éxito en la solución de problemas, la alta dedicación, y la ayuda a los pacientes y a los colegas. Ese modelo de prestigio contrasta con el que se percibe como dominante en la actualidad, basado fundamentalmente en la visibilidad pública y política.

El amplio y denso sistema de relaciones entre médicos (tanto de tipo profesional como de amistad) configura un "mundo social médico" ("mundo pequeño" "32-34 que facilita obviamente su ejercicio profesional a la vez que contribuye a la creación y mantenimiento de una identidad social propia. Este "pequeño mundo social" tiene además como una de sus características más importantes la cercanía entre las elites y el conjunto de la profesión (es decir la cercanía al poder) que permite una mayor comunicación entre los espacios de decisión y los profesionales. En conclusión podemos afirmar que el acceso a los espacios de toma de decisiones (profesionales y sanitarios) es rápido gracias al fuerte entramado de relaciones directas (de conocimiento personal) entre los profesionales y los altos cargos (de la profesión, de la Administración y de las organizaciones). El flujo de comunicación que se crea entre los profesionales y el poder asegura un mayor acceso de las problemáticas e intereses de los médicos a los espacios donde se toman decisiones sobre la forma y dinámica de su ejercicio. El mundo social médico es un "mundo pequeño" donde la distancia entre las elites y el conjunto de la profesión es considerablemente menor que en otras arenas sociales y políticas.

Los profesionales de las sociedades occidentales, especialmente los de servicios personales, destacan por posiciones ideológicas más liberales que la población general ${ }^{35}$. En concreto, los médicos barceloneses se sitúan en una posición ideológica media de centro izquierda (4,53 en una escala 0 izquierda 
10 derecha), más progresista que otras profesiones barcelonesas (abogados, arquitectos, ingenieros) ${ }^{36-37}$ y que la media poblacional ${ }^{38}$.

Los profesionales médicos destacan por su alta participación social, muy por encima de las medias de los ciudadanos del país ${ }^{39}$, ya sea como asesores en temas de salud (para organizaciones sociales y entes políticos) o como ciudadanos activo en organizaciones civiles. Aunque sus niveles de contribución y participación social son más altos, el modelo de participación coincide con el dominante en el país (aunque dándole un menor protagonismo al asociacionismo religioso) centrado en el deporte, la cultura y el arte, la religión y la acción caritativa. Paralela a su implicación social, los profesionales de la medicina colaboran activamente como expertos con las instituciones políticas de su entorno (Parlamento, gobiernos) en procesos de elaboración de políticas o acciones sanitarias. En definitiva la profesión médica realiza una contribución social muy destacada, más allá de su ejercicio, y se convierte en uno de los actores más relevantes de la sociedad civil.

Los procesos de cambio, internos y externos, llevan a los médicos a definir un proyecto de profesión para el siglo XXI que acentúa nuevas formas organizativas de la práctica y de la propia profesión y reafirma su orientación de servicio así como su contribución y papel social.

\section{AGRADECIMIENTOS}

A los miembros del Equipo de Investigaciones en Profesiones y Organizaciones: José L. C. Bosch, Alex Alarcón, Cecilia Borau, Julián Cárdenas, Fredes Insa y Christian Oltra.

\section{BIBLIOGRAFIA}

1. Freidson, E. El alma del profesionalismo. En Sánchez, M., Záez, J. y Svensson, L. Sociología de las Profesiones. Murcia. DM. 2003

2. Jovell, A. El futuro de la Profesión Médica. Barcelona. Biblioteca Josep Laporte. 2001.

3. Oriol Bosch, A. The health professions: Desirable futures. Barcelona. IES. 2000.

4. Rodríguez, J.A. Canvi social: la professió médica davant dels reptes d'una nova societat. En Quinzè Congrés de Metges i Biòlegs de Llengua Ctalana. Lleida. QCMBLC. 1996.

5. Equipo de Investigaciones en Profesiones y Organizaciones de la Universitat de Barcelona y Col.legi Oficial de Metges de Barcelona. Els Metges de Barcelona en el Nou Mil.leni. Barcelona. 2003.

6. Saks, M. Orthodox and Alternative Medicine. Londres. Continuum. 2003

7. Saks, M. Professions and the Public Interest: Professional Power, Altruism and Alternative Medicine. Londres. Routledge, 1995.
8. Krause, E.A. Death of the Guilds: Professions, States and the Advance of Capitalism, 1930 to the Present. New Haven. Yale University Press. 1996.

9. Rodríguez, J.A. The social transformation of the Spanish medical profession. En The Politics of Medicine in Spain. New Haven. Yale University. 1991.

10. Freidson, E. Professionalism: The Third Logic. Cambridge. Polity Press. 2001.

11. Pardell, H. ¿Tiene sentido hablar de profesionalismo, hoy?. Educación Médica v.6, n.2 (abr-jun, 2003).

12. Hafferty F. y Light, D. Professional Dynamics and the Changing Nature of Medical Work. Journal of Health and Social Behavior. Extra issue. 1995.

13. Johnson, T. Larkin G. y Saks M. (eds.). Health Professions and the State in Europe. Londres. Routledge, 1994.

14. Taylor, D. Changes in Society and in the Role of Doctors and the Medical Profession. Barcelona, 2001.

15. Rodriguez, J.A. Professions as networks. 5th European International Conference on Social Networks. Sitges, 1998.

16. Wolinsky, F.D. The professional dominance perspective revisited. The Milbank Quarterly. 1988, 66.

17. Wilensky, H.L. The professionalization of everyone?. American Journal of Sociology. 1964, 70.

18. Navarro, V. Professional dominance or proletarianization?: Neither. The Milbank Quarterly. 1988, 66.

19. Rodríguez J.A. y. de Miguel, J.M. Salud y Poder. Madrid. CIS. 1990.

20. Freidson, E. Profession of Medicine. Chicago, IL. The University of Chicago Press. 1970.

21. Larson, M.S. The Rise of Professionalism. Berkeley, CA. University of California Press. 1977.

22. Abbott, A. The System of Professions. Chicago, IL. The University of Chicago Press. 1998.

23. Freidson, E. Professionalism Reborn: Theory, Prophecy and Policy. Chicago. The University of Chicago Press. 1994.

24. Freidson, E. Professional Powers. Chicago. The University of Chicago Press.1986.

25. Salter, B. Medical Regulation and Public Trust: An International Review. Londres. King's Fund. 2000.

26. Oriol Bosch, A. Crisi del professionalisme o la solució per la crisis (www:iesalut.org/index.htm)

27. Wildes, K. Patient No More: Why did the golden age of medicine collapse?, America vol 185, 2001.

28. Taylor, D. Changes in Society and in the Role of Doctors and the Medical Profession. Barcelona, 2001.

29. Oriol Bosch, A. El desenvolupament integral dels professionals i dels serveis del sistema sanitari (www:iesalut.org/index.htm)

30. Freidson, E. Profession of Medicine. Chicago, IL. The University of Chicago Press. 1970.

31. Larson, M.S. The Rise of Professionalism. Berkeley, CA. University of California Press. 1977.

32. Watts, D.J. Small Worlds. Princeton, NJ. Princeton University Press, 1999.

33. Barabási, A-L. Linked. Nueva York. Plume Book. 2003

34. Buchanan, M. Nexus. Nueva York. Norton and Company. 2002.

35. Brint, S. In an Age of Experts: The Changing Role of Professionals in Politics and Public Life. Princeton, NJ. Princeton University Press. 1994.

36. Rodríguez, J.A. Encuesta de Profesiones y Organizaciones. Barcelona. UB. 1997.

37. Rodríguez Avila N. y Rodríguez, J.A. Cambios en la estructura y práctica de la profesión de abogado. En Sánchez, M., Záez, J. y Svensson, L. Sociología de las Profesiones. Murcia. DM. 2003.

38. World Value Survey 2000. http://www.worldvaluessurvey.org/ 39. World Value Survey 2000. http://www.worldvaluessurvey.org/ 